\title{
Fuzzy Control for Two-degree of Freedom Point Light Source Tracking System Based on Digital Signal Processor
}

\author{
Hongke Xu,Peichao Dong, Hao Chen,Hang Yan,Shan Lin \\ School of Electronic and Control Engineering \\ Chang'an University \\ Xi'an, 710064, China \\ e-mail: xuhongke@chd.edu.cn,726141442@qq.com
}

\begin{abstract}
In order to improve the efficiency of solar panels, this paper designs a DSP-based two-degree of freedom point light source fuzzy tracking system, which is based on the fuzzy reasoning technology and digital signal processor technology. It designs a photodiode based light source detection unit, and uses a two-degree of freedom steering gear PTZ as the execution unit. By analysis characteristics of the system and summing up the experience, it gave a set of fuzzy control rules, and determined some important control parameters. Considering the real-time control, and system stability, it uses the DSP as the core of the system control unit. Simulation and experiments results show the good performance of the system.
\end{abstract}

Keywords-point light source; track; fuzzy control ; steering gear PTZ; DSP

\section{INTRODUCTION}

With the depleting of the world energy, there are broad prospects for the development and utilization of renewable energy, which have become the focus of future energy. Solar energy as a clean and non-polluting energy, has very bright development prospects. Currently, most solar panels receive solar energy with fixed elevation angle and fixed position. The efficiency is extraordinarily low in such passive way to receive solar[1].It has become a hot research topic to improve the efficiency of solar receivers by actively adjusting the solar panels to track the sun's position[1 2], in recent years. There are two main methods to detect solar orientation now. The first one is calculating the azimuth and elevation angle by reference astronomical information[1]. The other one is to detect solar orientation through the sensor device[2 4]. And there are certain problems in both of these methods. The accuracy of the astronomical information calculated results is poor[1]. It is vulnerable to external causes such as the sun's rays change, which makes the system unstable[2]. Fuzzy control, based on fuzzy reasoning, is a nonlinear control strategy. It expresses the operating experience of skilled operator and common sense inference rules by fuzzy language. The precise mathematical model of the controlled object needs not to be known. It is not sensitive to changes in process parameters. It has strong robustness and a faster response and smaller overshoot[5 6].
This paper presents an implementation of the point light source tracking system, which can be applied to solar panels tracking the sun and realize the purpose of achieving maximum efficiency of absorb the solar energy. In this implementation, the differential signal of sensors is used as controlling basis, and the fuzzy algorithm is used as the core algorithm, which greatly improved the interference performance of the system. The TMS320LF2407A DSP chip, which is produced by TI Company, is used as the main control unit to enhance the real-time and stability of the system.

\section{THE STRUCTURE DESIGN OF POINT LIGHT SOURCE TRACKING SYSTEM}

The point light source tracking system can be decomposed into two sub-control systems, the horizon sub-control system and the vertical sub-control system. Both the two sub-control systems consist of signal acquisition unit, fuzzy control unit and execution unit. The system structure is shown in Figure 1.

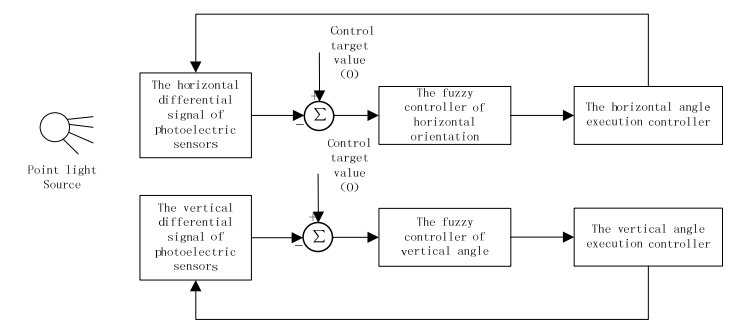

Figure 1. Point Light Source Fuzzy Tracking System Structure

\section{DESIGN OF SYSTEM HARDWARE}

The hardware of the system mainly contains central processing unit, signal measurement unit, control signal output unit, serial communication unit, keyboard unit and LCD unit. The hardware block diagram is shown in Figure 2. 


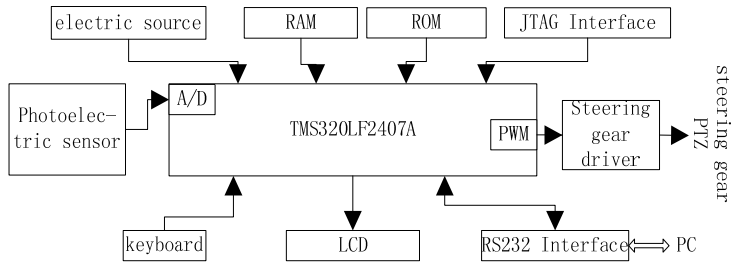

Figure 2. Block Diagram of System Hardware

\section{A. The central processing unit}

The core unit of the system is the TMS320LF2407A ultra-large-scale DSP chip, which is produced by the TI Company[8 9]. The high-performance static CMOS technology has been applied into the TMS320LF2407A, which makes the supply voltage of the chip reduce to $3.3 \mathrm{~V}$ and reduce the power consumption in a large part. The 40MIPS execution speed makes the instruction cycle shortened to $25 \mathrm{~ns}(40 \mathrm{MHz})$, which improve the real time control ability of the DSP. $32 \mathrm{~K}$ word flash (encryption), $2.5 \mathrm{~K}$ RAM and 500_ns A/D converter are integrated. On-chip event manager provides PWM interface and I/O function, which can meet a variety of motor driving function. Due to length limitation, circuit diagram not be given.

\section{B. The detection unit}

This paper uses the photosensitive diode as the detection device of point light source. The tube core of the photosensitive diode is photosensitive PN junction, which has the character of unilateral conductivity. Therefore, the photosensitive diode should apply reverse voltage when working. The photosensitive diode will cut off with very little saturated reverse leakage current, namely dark current, without light. When exposed to light, saturated leakage current increases greatly and contributes to the photocurrent, which change with the intensity of input light. Photosensitive diode has very good sensitivity to the change of the location of light source, and it can be used in real-time detection of point light source.

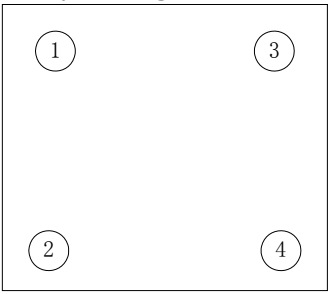

Figure 3. Location and Serial Number of Sensors

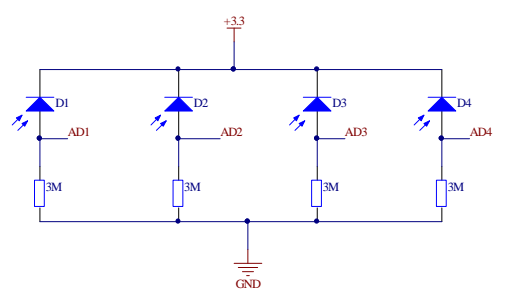

Figure 4. photoelectric sensor circuit diagram

The point tracking module designed in this paper comprises of four photosensitive diodes, which can be divided into two groups according their spatial location and each group have two diodes. The location and number of the four photosensitive diodes is shown in Figure 3, and the figure can be seen from the vertical angle to the sensor plane. The four photosensitive diodes cooperate with each other help to detect the point light source's position. As shown in Figure 4, the voltage applied to the photosensitive diodes is $3.3 \mathrm{~V}$ and the resistance value of divider resistance is $3 \mathrm{M}$, which ensure that the sensor output voltage within reference voltage range of the A/D converter used (0-3.3V).

\section{The execution unit}

The execution unit of the system this paper designed is a two-degree of freedom steering gear PTZ, which can be used to adjust the horizontal angle and vertical elevation. The rotation angle of the steering gear can be controlled by PWM signal. The PWM signal is generated by the event manager of TMS320LF2407A. The cycle of the PWM signal, which is used for controlling the steering gear, is $20 \mathrm{~ms}$. When the high level duration in a cycle of the PWM signal changes between $0.5-2.5 \mathrm{~ms}$, the rotation of the steering gear correspondingly change in the $0-180$ degrees. The Figure 5 shows the structure of the steering gear PTZ and the assembly of sensors.

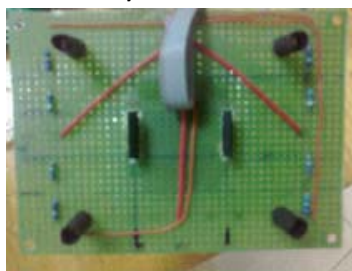

(Top view)

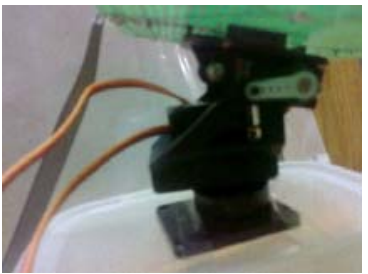

(Right view)
Figure 5. The Structure of the steering gear PTZ and the sensor assembly

\section{CONTROL ALGORITHM DESIGN}

\section{A. The system control structure}

The control theory of the system can be described as fallow. The horizontal deviation e1 can be calculated by subtracting the average output value of the right two sensors (No. 3 and No. 4) from average output value of the left two sensors (No. 1 and No. 2). The horizontal steering gear is controlled according to the value of e1.The greater the value of e 1 is and sharper the change rate of e 1 is, the faster the steering gear rotate. Similarly, the vertical deviation e2 can be calculated by subtracting the average output value of the up two sensors (No. 1 and No. 3) from average output value of the down two sensors (No. 2 and No. 4). The vertical steering gear is controlled according to the value of e2. Accordingly, the two dimensional fuzzy controlling structure is used in the point light tracking 
system. The control structure of the system is shown in Figure 6.

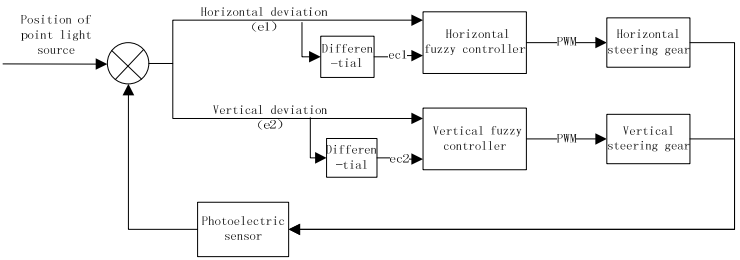

Figure 6. Control Structure of The System

\section{B. Fuzzy domain, fuzzy subset membership function}

Due to the machine and the circuit structure of the horizontal direction and the vertical direction is basically same, the fuzzy controller of horizontal and vertical direction use the same controlling structure. The fuzzy controller's input deviation (e) and deviation change rate (ec) have the same domain, which is [-3.3, 3.3]. The fuzzy subset of e and ec is \{negative big, negative small, zero, positive small, positive big\}, which is abbreviated as $\{\mathrm{NB}, \mathrm{NS}$, ZO, PS, PB\}. The domain of variation control output PWM signal $(\Delta u)$ is $[-6,6]$. The fuzzy subset of $\Delta u$ is \{negative big, negative small, zero, positive small, positive big\} abbreviated as $\{\mathrm{NB}, \mathrm{NS}, \mathrm{ZO}, \mathrm{PS}, \mathrm{PB}\}$, which is same with that of e and ec. The domain of $\Delta u$ is got by experience.

The member function of e, ec and $\Delta u$ is triangle-shaped membership function, as shown in the Figure 7. The triangle shape is narrow near the 0 , which is conducive to improve the sensitivity and restrain the overshoot. While, the triangle shape is wide far away from the 0 , in this case the error is large and the overshoot is hard to be caused.

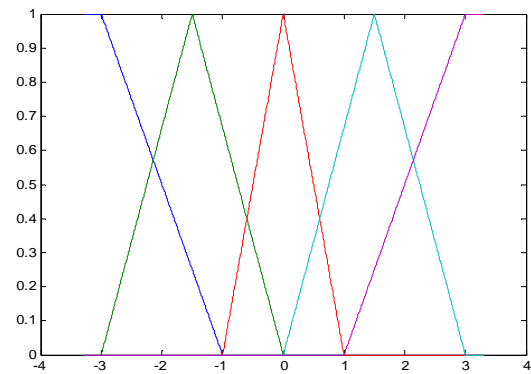

Figure 7. The Membership of Error, Error Change Rate and Output u

\section{Fuzzy control rules}

The established principle of the fuzzy control rule can be described as follow. When the deviation is large, the control output should mainly eliminate deviation as soon as possible. When the error is small, the control output should mainly prevent overshoot. If the ec is positive that means the position of the light source changes in positive direction and has a rising trend. If the deviation e is positive big at this time the variation of the steering gear control signal should be reduced. Conversely, the variation of the steering gear control signal should be increased. Through a comprehensive analysis of the experience of "experts", the established fuzzy rules table is listed in Table I .

TABLE I. THE FUZZY RULES

\begin{tabular}{|c|c|c|c|c|c|c|}
\hline \multirow{2}{*}{\multicolumn{2}{|c|}{$\Delta \mathbf{u}$}} & \multicolumn{5}{|l|}{ e } \\
\hline & & NB & NS & ZO & PS & PB \\
\hline \multirow{5}{*}{ ec } & NB & NB & NS & NS & NS & ZO \\
\hline & NS & NS & NS & NS & ZO & PS \\
\hline & ZO & NS & NS & ZO & PS & PS \\
\hline & PS & NS & ZO & PS & PS & PS \\
\hline & PB & ZO & PS & PS & PS & PB \\
\hline
\end{tabular}

D. Reasoning and defuzzification

The reasoning algorithm of this system is Mamdani fuzzy inference algorithm. The basic attribute set of the fuzzy inference system is show as follow. The collation operation uses minimum operation. The or operation uses maximum operation. The fuzzy implication uses minimal operation. Fuzzy rule synthesis uses maximum operation.

There are three commonly used defuzzification methods, including the max subjection degree method, center of gravity method and the weighted averages method. And the center of gravity method is the most reasonable, popular and attractive defuzzification method ${ }^{[4]}$. The center of gravity method is named for that it takes the center point of gravity of the fuzzy membership function curve and the horizontal axis formed area for the representative points. In theory, the center of gravity of a consecutive series of points in the output range should be calculated, namely:

$$
u=\frac{\int_{y} u_{c}(x) x d x}{\int_{y} u_{c}(x) d x}
$$

This method is complex, but it contains all the information of the output fuzzy subset, for which the center of gravity method is used in this paper.

\section{DESIGN OF THE SYSTEM SOFTWARE}

The main process of the software of the point light source tracking system is shown as follow. Firstly, collect the $A D$ values in interrupt mode, and process the $A D$ values with the mean filter method. Calculate the e and ec according to the filtered AD values. Secondly, fuzzify the values of $\mathrm{e}$ and $\mathrm{ec}$ with triangle-shaped membership function, which is introduced above, namely. Thirdly, calculate the membership degree of $\Delta u$ by searching the fuzzy rule table and doing fuzzy reasoning. Finally, defuzzify the $\Delta u$ with the center of gravity method, and use results of defuzzification to control the rotation of the steering gears. The main software process of the system is shown in Figure 8 , and the other interrupt service processes are abbreviated. 


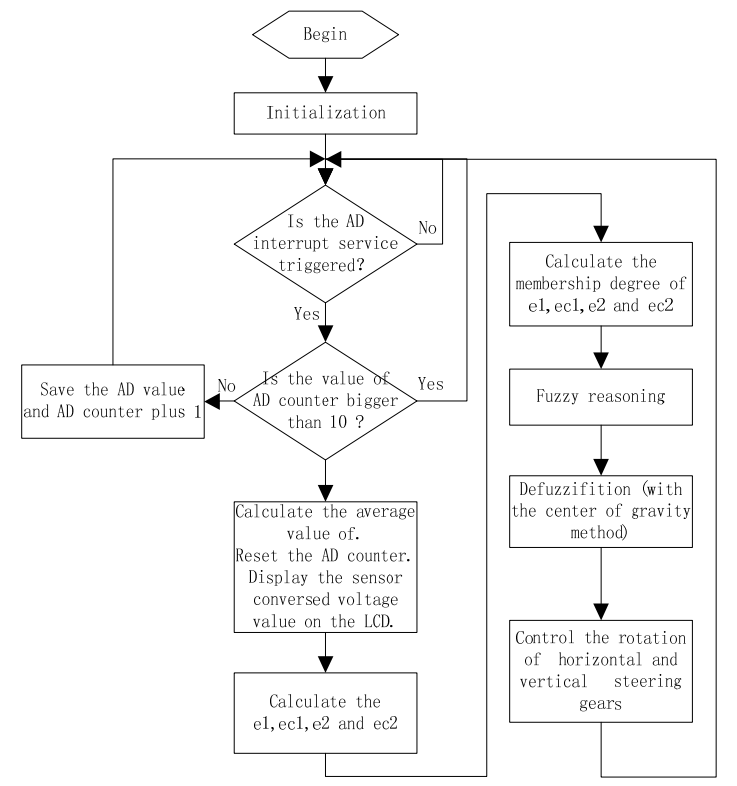

Figure 8. Main Process of System

\section{THE RESULTS OF SIMULATION AND EXPERIMENT}

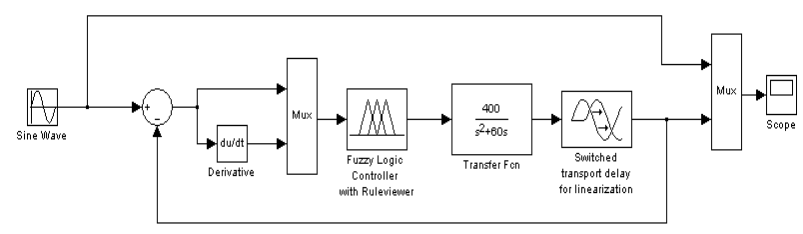

Figure 9. Simulation Structure

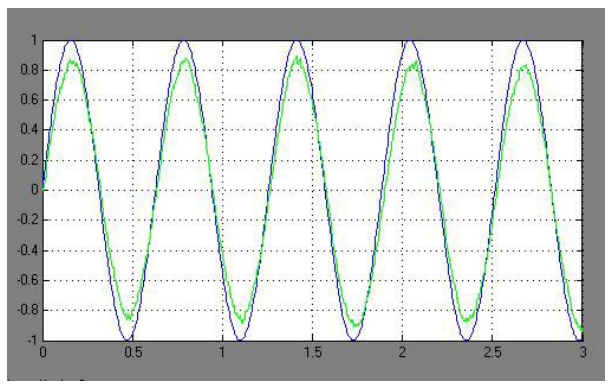

Figure 10. The Simulation Results

The structure of system fuzzy control algorithm simulation in Matlab is shown in Figure 9.The Figure 10 shows the result of the simulation. From the Figure 10, it can be seen that the system can accurately and quickly follow changes of input signal.

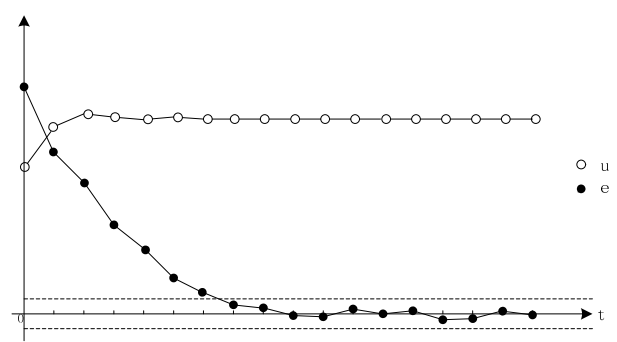

Figure 11. Step Response of System

The Figure 11 shows the step response of the system, in the figure the $u$ represents the control variable and the e represents error. From the Figure 11, it can be seen that the system has a good performance in response speed, maximum overshoot and control precision.

\section{SUMMARY}

This paper introduces a two-degree of freedom point light source fuzzy tracking system, which takes DSP as the core control unit. The design concept, control algorithms and designs of hardware and software are focused. The system introduced the fuzzy control algorithm, which solves the problem that the system model and control parameters are hard to be accessed. The real time of the system is ensured by the DSP, which has a high-speed calculation capability, being used to process data. The simulation results of the system show that the system has a good dynamic performance, which provides a good technical guidance to the design and operation system. The experimental data of actual system confirms the DSP based two-degree of freedom point light source fuzzy tracking system has good control performance.

\section{REFERENCES}

[1] Zhang Yi-chong, A DSP-Based Solar Tracking Control System[D], Shanghai Jiao Tong University,2008

[2] Yang Pei-huan, The research of high precision solar tracking sensor and Controller[D], Wuhan University of Technology,2010

[3] Wei Chun-hua,430 point light sources based tracking system microcontroller, HuNan Agricultural Machinery,2010(9),25-26

[4] Lei Dan, He Wei,Design of Spotlight Tracking System, Techniques of automation and Applications,2012(1),68-71

[5] Xi Ai-min, Fuzzy control technology[M], Xi'an Electronic and Science University press,2008

[6] Liu Jin-kun, Intelligent control[M], Electronic Industry Press,2010

[7] Pen Wei, Zhang Da-fa, Fuzzy Control for Steam Generator Water Level Based on Digital Signal Processor[J], Atomic Energy Science and technology,2010(9),283-286

[8] Zhang Dong-liang, DSP control theory and Application[M], China Machine Press,2011.

[9] TI. Digital signal processing solution for AC induction motor[R]. Texas, USA: Texas Instruments Incorporated, 2007. 